\title{
Splenic Abscess after Sleeve Gastrectomy: A Report of Two Cases
}

\author{
Nasser Sakran ${ }^{a} \quad$ Anat Ilivitzki $^{b} \quad$ Abdel-Rauf Zeinac ${ }^{c}$ Ahmad Assaliad \\ ${ }^{a}$ Department of General Surgery, Hillel Yaffe Medical Center, Hadera, ${ }^{b}$ Department of \\ Radiology, Rambam Health Care Campus, Haifa, 'Department of Radiology, Hillel Yaffe \\ Medical Center, Hadera, dDepartment of General Surgery, Rambam Health Care Campus, \\ Haifa, Israel
}

Key Words

Splenic abscess · Laparoscopic sleeve gastrectomy • Morbid obesity $\cdot$ Splenectomy

\section{Abstract}

Objective: Partial demarcation and infarction of the upper pole of the spleen is not a rare occurrence after laparoscopic sleeve gastrectomy (LSG). However, development of an abscess in the absence of leakage is extremely rare. Case Reports: We present herein two unusual case reports of splenic abscess in patients following LSG.

Copyright $(2012$ S. Karger GmbH, Freiburg

\section{Introduction}

Laparoscopic sleeve gastrectomy (LSG) is rapidly gaining momentum in bariatric surgery. Partial demarcation and infarction of the upper pole of the spleen following LSG is not uncommon and has been previously reported [1,2]. One of the steps in LSG is mobilization of the fundus including the division of the short gastric vessels (SGVs). In the vast majority of patients, it has no clinical relevance. On the other hand, formation of splenic abscess is an extremely rare consequence that has been never described before. Its rarity and the nonspecific clinical presentation could make the diagnosis difficult and could be easily confused with leakage and subdiaphragmatic abscess. vention.

We present herein two cases of splenic abscess after LSG managed by surgical inter- 
Fig. 1. A Axial and B coronal contrast-enhanced CT images through upper abdomen show multiple hypodense lesions (arrows) in the spleen (S) consistent with splenic abscesses, associated with mild splenomegaly. $\mathrm{K}$ denotes right kidney; L denotes liver.
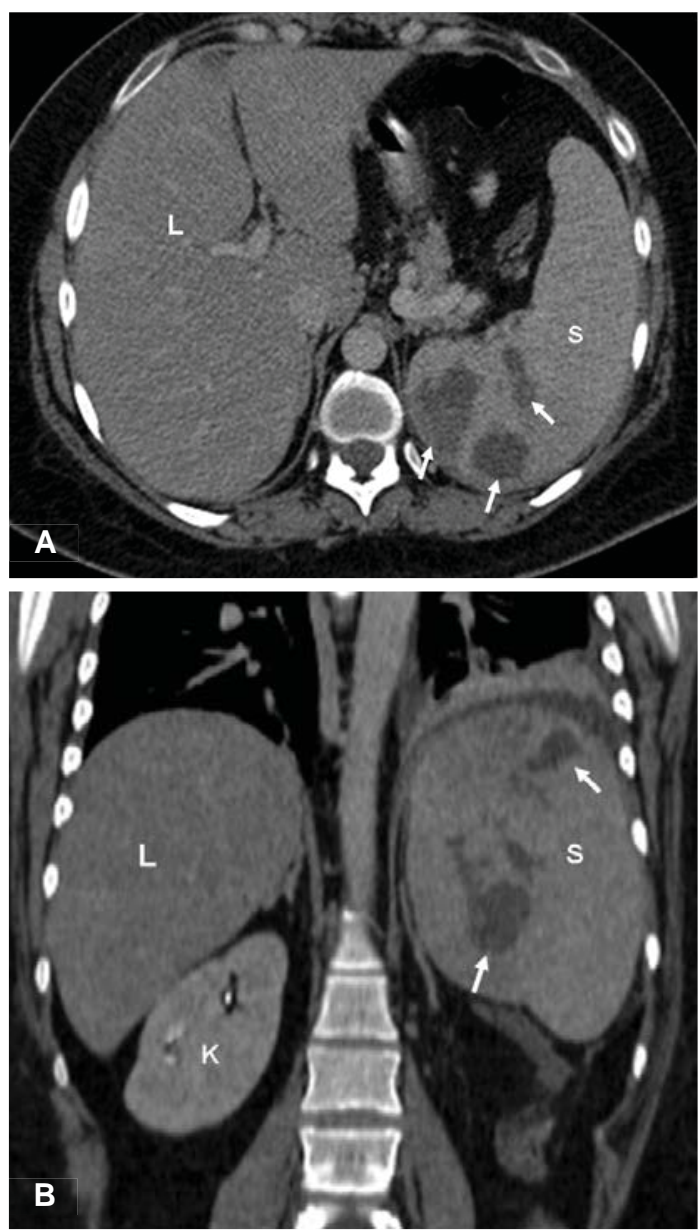

\section{Case Reports}

A retrospective review of the 750 cases that underwent LSG during the last 5 years in two surgical departments was undertaken. Two female patients with splenic abscess $(0.26 \%)$ were identified and analyzed. No intraoperative mishaps were identified in both cases, including possible conflict with the spleen. The diagnosis was made 6 and 8 weeks after surgery. The cultures revealed Streptococci in both cases. In one patient multiple splenic abscesses necessitated splenectomy.

Case 1

A 36-year-old obese female patient underwent LSG. No intraoperative mishaps were recorded and the immediate postoperative course was uneventful. She was discharged on postoperative day 4, and no findings or complaints were observed during the first postoperative visit (1 week after surgery). Two months later the patient reported persistent fevers, chills, and left upper quadrant (LUQ) abdominal pain of 2 weeks duration. There was no history of trauma or other associated illnesses. On physical exam the spleen was enlarged $3 \mathrm{~cm}$ below the left costal margin with localized tenderness in the LUQ. Laboratory investigation revealed elevated white blood cells (WBCs) of 20,000 with a left shift. Abdominal computed tomography (CT) scan showed multiple low-density lesions in an enlarged spleen, $17 \times 14 \times 6 \mathrm{~cm}$ (fig. 1a,b). These findings were consistent with diagnosis of splenic abscess. Echocardiography was normal. The patient was initially managed nonoperatively with parenteral broad-spectrum antibiotics, but the fever and pain persisted. Therefore, she was managed by open splenectomy, due to enlarged spleen. The findings included remarkably enlarged spleen with multiple inflammatory adhesions involving the diaphragm, parietal peritoneum, greater omentum, and left lobe of the liver. The gastric sleeve was not 
Fig. 2. Contracted enhanced CT in coronal plain. Air containing splenic abscess in the cranial portion of the spleen, with air babble protruding into subphrenic space. Small amount of pleural effusion can be seen on the left.

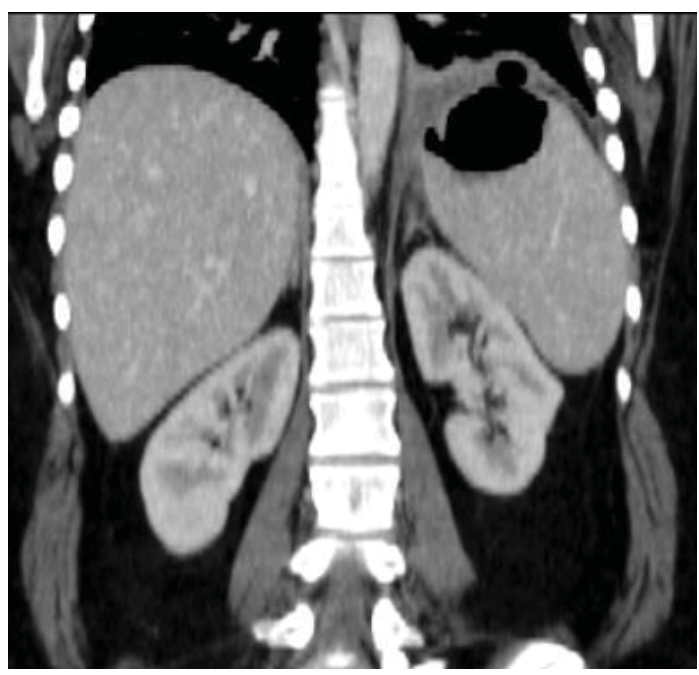

Fig. 3. Fluoroscopy. Contrast media injected through percutaneous drain positioned in splenic abscess. Note contrast enhancement of the splenic vein and the portal vein (small arrows).

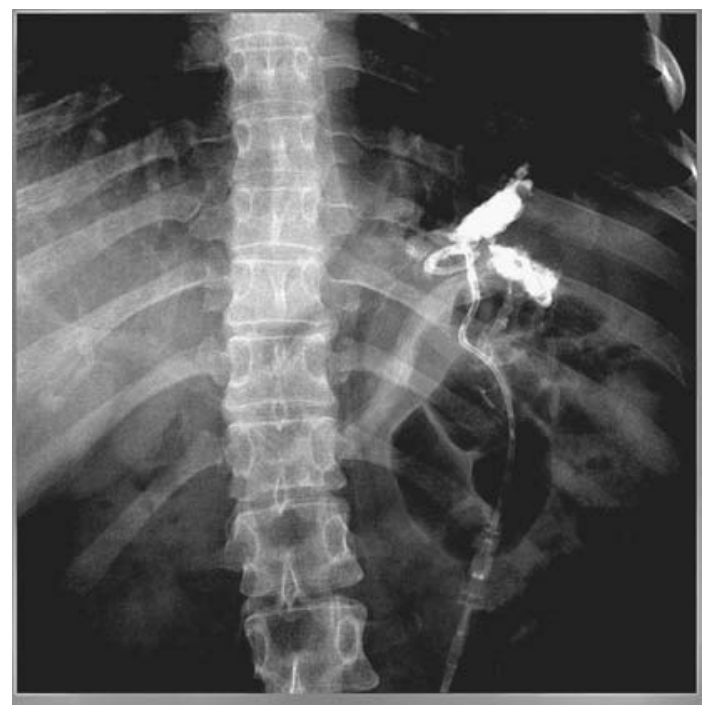

involved and looked normal. Cultures y ielded Streptococcus spp., Escherichia coli E.S.B.L (extended spectrum beta lactamase), and Enterococcus faecalis. Pathologic examination showed multiple abscesses with focal necrosis with widespread fibrosis. Recovery was uneventful.

Case 2

A 35-year-old female obese patient underwent LSG. 75 days later, the patient was admitted to the hospital due to LUQ abdominal pain, left shoulder pain, and fever. On physical examination the patient had flank and abdominal tenderness in the left upper abdomen. Routine laboratory testing revealed a mild leukocytosis. Abdominal CT scan of the abdomen showed an abscess of the upper pole of the spleen in close proximity to the left diaphragm, with air-fluid level of $5.3 \mathrm{~cm}$ (fig. 2). Gastroscopy and UGI test revealed no evidence for leakage. The abscess was drained percutaneously and the patient was managed with broad-spectrum antibiotics. Due to lack of resolution of the abscess, a fistulography was performed which ruled out leakage and showed that the process was intrasplenic with demonstration of the portal system (fig. 3).

The patient underwent a diagnostic laparoscopy 3 months later due to failure of percutaneous drainage. During the exploration, the gastric sleeve looked normal with no evidence for contact with the 
abscess. The abscess was drained laparoscopically, and a transabdominal drain was placed. The culture from the abscess yielded Staphylococcus spp., Enterobacter cloacae, and Streptococcus mitis and oralis. The postoperative course was uneventful, and the drain was removed 2 weeks from surgery. The patient remained asymptomatic ever since.

\section{Discussion}

Splenic abscess may develop after generalized infection, hematological disorder, and trauma. It occurs due to bacteremic seeding of a previously infarcted area or by direct seeding of the spleen from an adjacent infectious process. Predisposing conditions for primary abscesses include diabetes mellitus, malignancy, polycythemia vera, endocarditis, previous trauma, sickle cell disease, urinary tract infection, intravenous drug abuse, AIDS, and other immune-compromised conditions [3-5]. It has been seldom reported following gastric surgery including Nissen fundoplication and gastrectomy for cancer [6].

Cultures from splenic abscess usually are polymicrobial and include Gram-positive bacteria, mycobacteria, fungi, and anaerobes [7-9]. The common signs and symptoms of splenic abscess are nonspecific. CT scan remains the gold standard for definitive diagnosis [5], but ultrasound can effectively demonstrate many of the characteristics of splenic abscess. Both of these imaging studies have a sensitivity of $98 \%[10,11]$. Classical teaching advocated splenectomy with antibiotics as it removes the complete focus of infection [10, $12,13]$. However, recent evidence has shown increasing use of laparoscopic or percutaneous drainage of splenic abscess as an alternative, in order to preserve splenic function, especially in a solitary splenic abscess. This category has a favorable outcome after treatment with intravenous antibiotics and CT-guided or ultrasound-guided percutaneous drainage. This in contrast to multiple abscesses, which most commonly require splenectomy $[12,14]$. In these cases and in cases where percutaneous drainage fails, laparoscopic or open drainage is an option, thereby avoiding splenectomy [9].

Splenic demarcation and infarction of the upper pole is not uncommon and has been reported following LSG. Division of the SGVs during LSG may lead to splenic infarction. In the vast majority of patients, it has no clinical relevance $[15,16]$. To the best of our knowledge, formation of an abscess is an extremely rare occurrence and has never been reported. The clinical presentation is not specific and could be easily confused with leakage and subdiaphragmatic abscess. We postulate that later infection of the splenic infarct, either via direct extension, from a nearby infection, or hematogenically is the possible mechanism for its occurrence. In our cases, an adjacent infection was ruled out as no leakage was demonstrated and postoperative course was uneventful. The abscesses became symptomatic in the intermediate period following surgery. Temporary immune suppression in the immediate postoperative period associated with rapid weight loss and limited oral intake, could possibly contribute to the formation of the abscesses in a partially infarcted spleen from a transient bacteremia.

In conclusion, splenic abscess should be taken into account as a rare complication in patients undergoing LSG. Every case presenting such a complication should undergo a complete workup in order to rule out possible leakage.

\section{Disclosure Statement}

The authors have no commercial associations that might be a conflict of interest in relation to this article. 
Sakran et al.: Splenic Abscess after Sleeve Gastrectomy: A Report of Two Cases

\section{References}

1 Stamou KM, Menenakos E, Gomatos IP, Panousopoulos SG, Smparounis S, Leandros E, Zografos G: Clinical implications of sleeve gastrectomy as a source of spleen infarction or ischemia. Obes Surg 2011;21:14901493.

2 Dhanasopon AP, Lewis CE, Folek JM, Dutson EP, Mehran A: Splenic infarct as complication of sleeve gastrectomy. Surg Obes Relat Dis 2009;5:626-629.

3 Ramakrishnan MR, Sarathy TKP, Balu M: Percutaneous drainage of splenic abscess: case report and review of literature. Pediatrics 1987;79:1029-1030.

- 4 Aessopos A, Politou M, Farmakis D, Sideris P, Grapsa A, Andreopoulos A, Kalahanis N, Karagiorga M: Staphylococcus aureus abscess of the spleen in a beta-thalassemia patient. Scand J Infect Dis 2002;34:466-480.

5 Smyrniotis V, Kehagias D, Voros D, Fotopoulos A, Lambrou A, Kostopanagiotou G, Kostopanagiotou E, Papadimitriou J: Splenic abscess. An old disease with new interest. Dig Surg 2000;17:354-357.

- 6 Martínez DG, Sánchez AW, García AP: Splenic abscess after laparoscopic Nissen fundoplication: a consequence of short gastric vessel division. Surg Laparosc Endoscopic Percutan Tech 2008;18:82-85.

7 Bessho H, Ichihara I, Takii M: A case of splenic abscess due to Chlamydia pneumonia. Diag Microbiol Infect Dis 2001;39:261-264.

- 8 Colmenero Jde D, Queipo-Ortuño MI, Maria Reguera J, Angel Suarez-Muñoz M, Martín-Carballino S, Morata P: Chronic hepatosplenic abscesses in Brucellosis. Clinico-therapeutic features and molecular diagnostic approach. Diagn Microbiol Infect Dis 2002;42:159-167.

9 Phillips GS, Radosevich MD, Lipsett PA: Splenic abscess: another look at an old disease. Arch Surg 1997;132: 1331-1335.

10 Ooi LL, Nambiar R, Rauff A, Mack PO, Yap TL: Splenic abscess. Aust N Z J Surg 1992;62:780-784.

11 Chang KC, Chuah SK, Changchien CS, Tsai TL, Lu SN, Chiu YC, Chen YS, Wang CC, Lin JW, Lee CM, Hu TH: Clinical characteristics and prognostic factors of splenic abscess: a review of 67 cases in a single medical center of Taiwan. World J Gastroenterol 2006;12:460-464.

12 Cabonell AM, Kercher KW, Matthews BD, Joels CS, Sing RF, Heniford BT: Laparoscopic splenectomy for splenic abscess. Surg Laparosc Endosc Percutan Tech 2004;14:289-291.

13 Paris S, Weiss SM, Ayers WH Jr, Clarke LE: Splenic abscess. Am Surg 1994;60: 358-361.

14 Ng KK, Lee TY, Wan YL, Tan CF, Lui KW, Cheung YC, Cheng YF, et al: Splenic abscess: diagnosis and management. Hepatogastroenterology 2002;49:567-571.

15 Nores M, Phillips EH, Morgenstern L, Hiatt JR: The clinical spectrum of splenic infarction. Am Surg 1998; 64: 182-188.

16 Antopolsky M, Hiller N, Salameh S, Goldshtein B, Stalnikowicz R: Splenic infarction: 10 years of experience. Am J Emerg Med 2009;27: 262-265. 